\title{
Functional Outcome Scores With Standard Myoelectric Prostheses In Below-Elbow Amputees
}

\author{
Stefan Salminger, MD $\mathrm{PhD}^{1,2}$, Ivan Vujaklija, $\mathrm{PhD}^{3,4}$, Agnes Sturma, $\mathrm{MSc}^{2,5}$, \\ Timothy Hasenoehrl, $\mathrm{MSc}^{6}$, Aidan D. Roche, MD, $\mathrm{PhD}^{2,7}$, Johannes A. Mayer, $\mathrm{MD}^{2}$, \\ Laura A Hruby, $\mathrm{MD}^{2,6}$ and Oskar C. Aszmann, $\mathrm{MD}{ }^{1,2}$
}

${ }^{1}$ Division of Plastic and Reconstructive Surgery, Department of Surgery, Medical University of Vienna, Waehringer Guertel 18-20, Vienna, Austria

${ }^{2}$ Christian Doppler Laboratory for Restoration of Extremity Function, Medical University of Vienna, Waehringer Guertel 18-20, Vienna, Austria

${ }^{3}$ Department of Bioengineering, Imperial College London, Kensington, SW7 2AZ, UK

${ }^{4}$ Clinic for Trauma Surgery, Orthopedic Surgery and Plastic Surgery, Research for

Neurorehabilitation Systems, University Medical Center Goettingen, Robert-Koch-Straße 40, 37075 Goettingen, Germany

${ }^{5}$ Master Degree Program “Health Assisting Engineering”, University of Applied Sciences FH Campus Wien, Favoritenstraße 226, 1100 Vienna, Austria

${ }^{6}$ Department of Physical Medicine and Rehabilitation, Medical University of Vienna, Waehringer Guertel 18-20, Vienna, Austria

${ }^{7}$ Department of Plastic Surgery, Southmead Hospital, North Bristol NHS Trust, Southmead Road, Westbury-on-Trym, Bristol UK 


\section{Correspondence:}

Oskar C. Aszmann, MD

Div. of Plastic and Reconstructive Surgery

Dept. of Surgery, Medical University of Vienna

Waehringer Guertel 18-20

A-1090 Vienna, AUSTRIA

Tel.: 0043140400 69940; Fax.: 004314040069880

Email: oskar.aszmann@meduniwien.ac.at

\section{Authors Disclosures:}

The authors have no competing interests to declare. This work was supported by the Christian

Doppler Research Foundation a subdivision of the Austrian Federal Ministry of Economy, Family and Youth, the Austrian Council for Research and Technology Development. 


\section{Abstract \\ Objective}

To report normative outcome data of prosthetic hand function in below elbow amputees using four different objective measurements closely related to activities of daily living (ADL).

\section{Design}

Seventeen patients who underwent prosthetic fitting after unilateral below-elbow amputation were enrolled in this study. Global upper extremity function was evaluated using the Action Research Arm Test (ARAT), Southampton Hand Assessment Procedure (SHAP), the Clothespin-Relocation Test (CPRT) and the Box and Block Test (BBT), which monitor hand and extremity function.

\section{Results}

The patients achieved a mean ARAT score of $35.06 \pm 4.42$ of 57 . The average SHAP score was $65.12 \pm 13.95$ points. The mean time for the CPRT was $22.57 \pm 7.50$ seconds and the mean score in the BBT was $20.90 \pm 5.74$.

\section{Conclusions}

In the current economic situation of health care systems, demonstrating the effectiveness and necessity of rehabilitation interventions is of major importance. This study reports outcome data of below-elbow amputees and provides a useful guide for expected prosthetic user performance.

\section{Key words}

Upper limb, amputation, prosthetic rehabilitation, outcome measurement 


\section{Introduction}

The advancement of multifunctional hand prostheses, novelties in prosthetic control strategies and new surgical techniques have drawn much attention to the evaluation of functional outcomes among upper limb prostheses users. ${ }^{1-4}$ Outcome measurements include survey or questionnaire based evaluations as well as rated or timed assessments quantifying satisfaction with the device, pain, prosthesis use or effectiveness of prosthetic training. ${ }^{1,5-9}$ However, the literature is lacking standard outcome data of below-elbow amputees gathered with objective, timed and non-rater dependent outcome measurements scoring prosthetic hand function closely related to activities of daily living.

Over the last decades, a great variety of hand function measurements have been established to evaluate the functional outcome or manual dexterity after hand surgical therapies or prosthetic rehabilitation. ${ }^{1,10-12}$ Still, defining success of prosthetic rehabilitation in upper limb amputees remains a difficult task, especially as compared to lower limb amputees, where functional gait analysis is mostly accepted as the main outcome measure. ${ }^{13}$

The use of validated objective outcome measurements is important, as the conclusions drawn from this functional outcome data may influence future prosthetic service, health care policy and prescribing of prosthetic devices in upper limb amputees. ${ }^{1,14}$ The emphasis on outcome measurements across prosthetic rehabilitation of the last years shows that standardized outcomes are essential for the development and maintenance of an effective health care system. $^{15}$

The growing number of outcome measures for prosthetic users increases the difficulty for the clinician to choose the most suitable evaluation tools. Objective functional tests closely related to activities of daily living are the minority. At our institution we use four different 
objective and timed functional measurements to evaluate gross and fine manual dexterity of upper limb amputees using prosthetic devices. The Southampton Hand Assessment Procedure (SHAP) represents an objective outcome measure where most tasks are related to activities of daily living. Additionally, the Box and Blocks Test (BBT) as well as the ClothespinRelocation Test (CPRT) were performed to evaluate repeatability and dexterity in detail, whereas the Action Research Arm Test (ARAT) gives an insight into full arm motion.

One of the measures recommended by experts in the field and widely used in specialist upper limb clinics is the Assessment of Capacity for Myoelectric Control (ACMC). ${ }^{9}$ Although the ACMC represents a validated assessment tool specifically designed for upper limb amputees using myoelectric devices, it can be considered as subjective as it depends on an observers' rating. ${ }^{16}$ Additionally the observers need extensive training to use this measure and rate accordingly. ${ }^{17}$ This is also true for the Activities Measure for Upper Limb Amputees (AMULA). ${ }^{18}$ Therefore these tests were not included in the outcome measures reported here, although they provide important information about real-world functional capabilities.

Here we present normative functional outcome data of seventeen unilateral below-elbow amputees using four different objective and timed assessment tools. The need for such standard norm data gathered by objective outcome measures has been highlighted by experts in this field. ${ }^{15,19}$

\section{Material and methods}

\section{Patients}

Seventeen patients who underwent prosthetic fitting after unilateral below-elbow amputation were enrolled in this observational study to evaluate the functional outcome of prosthetic rehabilitation. All patients were enrolled from the special outpatient hand clinic. All patients 
gave written informed consent to take part in this study, which was reviewed and approved by the local Institutional Review Board. Patients were eligible if they were at least 18 years old, suffered a unilateral below elbow amputation and used their latest fitted myoelectric prosthesis for at least one year. Patients were excluded if they had significant uncorrectable visual deficits, major communication or neurocognitive deficits.

\section{Outcome measurements}

Global upper extremity function was evaluated using the Action Research Arm Test $(\mathrm{ARAT})^{20}$, Southampton Hand Assessment Procedure (SHAP) ${ }^{21}$, the Clothespin-Relocation Test $(\mathrm{CPRT})^{11}$ and the Box and Block Test $(\mathrm{BBT})^{22}$. Additionally, patients were asked about their average wearing time of the prosthetic device per day.

The ARAT is an observational test used to determine upper limb motor function and has been validated for use with patients with cognitive impairment of hand control. ${ }^{20}$ It consists of 4 sections with different tasks and a maximum of 57 points attainable. ${ }^{20}$ The ARAT was performed according to the standardized approach from Yozbatrian et al. ${ }^{23}$

The SHAP is a clinically validated hand function test and was developed to assess the effectiveness of upper limb prostheses. ${ }^{21}$ It is made up of 6 abstract objects (both heavy and light variations) and 14 Activities of Daily Living (ADL) with each task timed by the participant themselves. Normal hand function is regarded as 100 points in the SHAP. ${ }^{12}$

The CPRT is an adapted clinical test from the Royal Graded Pinch Exerciser ${ }^{11}$ that involved transferring four clothespins of various strengths from a horizontal bar to a vertical one. This test is particularly useful to assess rotation, flexion and extension movements at the wrist. The mean time of three repetitions is calculated. 
The BBT is suggested as a measure of gross manual dexterity. ${ }^{22}$ It is made up of a wooden box divided into two compartments, one filled with 100 blocks. The BBT score is equal to the number of cubes transferred from one compartment to another in one minute. ${ }^{22}$ Again, the mean of three repetitions is calculated.

All outcome measures (ARAT, BBT, CPRT, SHAP) were assessed by the same experienced physical therapist for all subjects. The different outcomes measures were performed within one visit. Assessment started with the SHAP and was continued with the other measures after a break of 15-30 minutes. In case of fatigue the patients had the opportunity to take a break at any time during the entire assessments.

\section{Statistical methods}

All outcome assessments used categorical variables. A two-tailed Mann-Whitney-U-test as well as Pearson correlation test were used for the analysis, with an alpha level of 0.05 . In regards to the Mann-Whitney-U-test, patients were grouped in amputees with dominant or non-dominant hand loss. The null hypothesis for each test was that the mean of the population from which the samples were taken, was the same for both groups. Equality of variances was not assumed. A correction for multiple comparisons was not performed, as the main goal is to present norm data and the sample size limits statistical interpretation.

\section{Results}

\section{Patient demographics}

Patient demographics are documented in Table I. The mean patient age at the time of amputation was $26.12 \pm 11.20$ years. One female and sixteen male patients were included. These patients sustained unilateral amputation of the hand at below-elbow level due to work related traumatic injuries $(n=15)$, tumor related amputation $(n=1)$ and congenital hand 
agenesis $(n=1)$. Twelve patients sustained amputation of their dominant hand, and five of the non-dominant hand. At the time of functional assessment patients had an experience using two-signal myoelectric control between one and 16 (mean 6.76) years.

Patients used their existing devices and sockets for the assessments. They were fitted with commercially available prosthesis from Otto Bock HealthCare Products GmbH, Germany (SensorHand Speed ( $n=8)$, Michelangelo Hand ( $n=5)$, Transcarpal Hand DMC Plus $(n=3)$ ) or Bebionic hand from Steeper Manufacturing Centre, Leeds, United Kingdom ( $n=1)$. All patients used two-signal direct myoelectric control. Some patients $(n=4)$ also used other types of myoelectric devices for specific tasks on an irregular basis at home. None of them used body-powered devices.

\section{Assessment procedure}

All patients were able to complete the four outcome measurements at one visit. The SHAP was performed first and took between 25 and 60 minutes. After the mandatory break following the SHAP, the majority of the patients did not ask for an additional pause. None of the patients reported fatigue during the different assessments. None of the other tests required more than 15 minutes of time.

\section{Functional outcome}

All functional outcomes are documented in Table II. The investigated patients achieved a mean ARAT score of $35.06 \pm 4.42$ out of 57 . The mean blocks moved in the BBT was 20.90 \pm 5.74 , and the mean time for fulfilling the CPRT was $22.57 \pm 7.50$ seconds. The average SHAP score was $65.12 \pm 13.95$ points. The patients use their prosthesis for an average of $11.88 \pm 2.47$ hours per day. 
There were no significant differences in ARAT $(p=0.790), \operatorname{BBT}(p=0.170), \operatorname{CPRT}(p=0.673)$ or SHAP ( $\mathrm{p}=0.958)$ between patients with dominant or non-dominant hand loss. Additionally, there were no significant correlations between prosthetic experience and ARAT $(p=0.546)$, BBT $(p=0.387)$, CPRT $(p=0.459)$ or SHAP $(p=0.527)$, or mean daily wearing time of the prosthetic device $(\mathrm{p}=0.810)$. There were no significant correlations between the daily wearing time or age of the patient (at investigation and at amputation) and all objective outcome measurements. However, there was a significant correlation between the age of the patient at investigation and the daily wearing time $(\mathrm{p}=0.037)$ showing younger patients wearing their prosthetic device longer. Additionally, there was a significant correlation between the SHAP scores and the CPRT $(\mathrm{p}=0.002)$, the BBT $(\mathrm{p}=0.002)$ and the ARAT $(\mathrm{p}=0.000)$ confirming the validity of the SHAP test. Still, even the simple BBT correlates mostly significant with the SHAP $(\mathrm{p}=0.002)$, the CPRT $(\mathrm{p}=0.004)$ and the ARAT $(\mathrm{p}=0.011)$ documenting the high predictability of this outcome measure. The correlation between CPRT and ARAT was not significant $(\mathrm{p}=0.060)$. Additionally, there were no significant differences in ARAT $(p=0.243)$, BBT $(\mathrm{p}=0.486)$, CPRT $(\mathrm{p}=0.758)$ or SHAP $(\mathrm{p}=0.142)$ between patients using SensorHand Speed $(n=8)$ or Michelangelo Hand $(n=5)$, which were the most commonly used prosthetic devices within this study.

\section{Discussion}

This study reports outcome data of below-elbow amputees with well-known functional assessment tools. Objective outcome measurements in upper limb prosthetic users are essential to evaluate the effectiveness of different prosthetic devices, the impact of new surgical techniques and the patients' capabilities. ${ }^{14,16}$ The International Society for Prosthetics and Orthotics (ISPO) has recently established a working group on upper limb outcome measures. ${ }^{14}$ This fact clearly shows the need for a meaningful systematic functional evaluation of upper limb amputees, as well as reporting standard outcome data. High costs for 
myoelectric devices have to be justified in health care systems and functional benefits should be quantifiable and compared to a norm sample. ${ }^{14}$ As stated by Bouwsema et al., time represents a key parameter in prosthesis use. ${ }^{12}$ Therefore objective and timed tests for the assessment of upper limb prosthetic function were used. The presented outcomes of seventeen below-elbow amputees should serve as norm data set, which can be useful for setting realistic goals for rehabilitation.

The SHAP was designed to determine the effectiveness of terminal prosthetic devices as well as the users' ability to control it by focusing on unilateral performance. ${ }^{8}$ It is also recommended by the Upper Limb Prosthetic Outcome Measures group and has shown to be a good measure of prosthetic user's skill level. ${ }^{12,24}$ While the patient is instructed on the different tasks by an evaluator, he/she times each task himself and the need for subjective opinion of an assessor is eliminated. ${ }^{8}$ As such, this evaluation tool can be used easily for outcome measurements among different groups to establish normative scores of a large cohort of upper limb amputees. ${ }^{25}$ Additionally, the SHAP takes only about 30-40 minutes in experienced prosthetic users and is a self-contained portable unit, therefore appropriate for clinical use. Moreover, this data set has shown strong significant correlation of the SHAP scores with the other outcome measures, indicating good predictability of this test. However, due to the multiple restrictions within the test protocol, similarity to real activities of daily living can be discussed and a measure of quality of movement is not provided. Therefore the SHAP can be considered as good test to evaluate prosthetic function, while other tests (ACMC, AMULA) might be more useful to evaluate the International Classification of Function (ICF) domain of activity. These rated tests provide functional data including quality of movement outside of a highly controlled, artificial setup. Future outcome research may include a larger cohort and investigate if assessments for function activity and participation correlate. (ACMC, AMULA, DASH) 
The ARAT is similar to the SHAP as evaluating hand function by simulating daily life activities, however, compared to the SHAP evaluates also elbow and shoulder function particularly. The ARAT is also frequently used to evaluate and monitor function of transplanted hands. ${ }^{25-27}$ Especially with multifunctional prosthetic devices it is important to involve different sequential functions in one task. Therefore, CPRT and BBT were chosen to monitor improvements in control strategies especially; they are widely used in the clinical setting and thus are familiar to hand therapy clinicians.

Although the SHAP includes some tasks requiring bimanual activities, the importance of bimanual dexterity, especially in unilateral amputations, is still underestimated. As the ARAT was originally not designed to evaluate prosthetic hand function, time limits for maximum scores as proposed by Yozbatrian et al ${ }^{23}$ are only reached by the most skilled prosthetic users, and therefore cause a floor effect. This can be seen by the small standard deviation of 4.42 points in the ARAT scores within the presented cohort. Still, the ARAT scores strongly correlate with the SHAP $(\mathrm{p}<0.001)$, the CPRT $(\mathrm{p}=0.060)$ and the BBT $(\mathrm{p}=0.011)$ supporting the use also in the assessment of prosthetic hand function. As performing all four assessments takes a considerable amount of time and the single tests show high correlations within this study, it does not seem to be necessary to perform multiple tests for outcome evaluation. Although fatigue was not reported by the patients included in this study, this still might be an issue using multiple timed outcome measures.

Although different prosthetic devices were used within this study, no significant differences between the outcomes measurements and the terminal devices could be seen. All patients used a two-signal direct control and the devices had the same basic functions. It was not the goal to distinguish between functional capacity of the different prosthetic devices, as the patient number was too low. 
In most studies, the length of daily prosthetic use indicates the success of amputee rehabilitation. ${ }^{13}$ However, different surveys have shown, that some patients do wear their myoelectric device only as a cosmetic prosthesis. ${ }^{6,7}$ Kejlaa ${ }^{28}$ proposed that wearing the prosthesis for more than eight hours is an indication for success, less than eight hours only a partial success. ${ }^{28}$ As shown in this study, younger patients seem to wear their prosthetic device longer, albeit the sample size of seventeen patients limits statistical interpretation. Additionally, no significant correlation could be seen between the prosthetic wearing time and functional outcome scores. Although high functionality of the device and likewise high scores in functional evaluation do not predict the actual use in daily life, objective outcome measurements are important to monitor hand function over time as well as depict achievements of new technologies. Thus, overall success of prosthetic rehabilitation should be based on a combination of objective function, rated performance in daily-life activities, wearing time, patient satisfaction and participation. ${ }^{13}$ As concluded by different working groups, this will not be possible with one single standard measure, but with a combination of assessment tools evaluating function and activities as well as questionnaires for reporting participation and quality of life.

\section{Conclusion}

In the current economical situation of health care systems, demonstrating the effectiveness and necessity of rehabilitation interventions is of major importance. Here we report normative outcome data of below-elbow amputees with standard myoelectric devices using validated standardized objective measurement tools. This data set should help therapeutic teams as well as the prosthetic service providers to gauge rehabilitative success or failure of their prosthetic care. 


\section{References}

1. Lindner HYN, Nätterlund BS, Hermansson LMN. Upper limb prosthetic outcome measures: review and content comparison based on International Classification of Functioning, Disability and Health. Prosthet Orthot Int. 2010;34(2):109-128. doi:10.3109/03093641003776976.

2. Kuiken TA, Dumanian GA, Lipschutz RD, Miller LA, Stubblefield KA. The use of targeted muscle reinnervation for improved myoelectric prosthesis control in a bilateral shoulder disarticulation amputee. Prosthet Orthot Int. 2004;28(3):245-253. http://www.ncbi.nlm.nih.gov/pubmed/15658637. Accessed August 10, 2014.

3. Pasquina PF, Evangelista M, Carvalho AJ, et al. First-in-man demonstration of a fully implanted myoelectric sensors system to control an advanced electromechanical prosthetic hand. Journal of Neuroscience Methods. 2014.

4. Salminger S, Roche AD, Hruby LA, et al. Prosthetic reconstruction to restore function in transcarpal amputees. J Plast Reconstr Aesthet Surg. 2015. doi:10.1016/j.bjps.2015.10.029.

5. Pezzin LE, Dillingham TR, Mackenzie EJ, Ephraim P, Rossbach P. Use and satisfaction with prosthetic limb devices and related services. Arch Phys Med Rehabil. 2004;85(5):723-729. http://www.ncbi.nlm.nih.gov/pubmed/15129395.

6. Datta D, Selvarajah K, Davey N. Functional outcome of patients with proximal upper limb deficiency--acquired and congenital. Clin Rehabil. 2004;18(2):172-177. http://www.ncbi.nlm.nih.gov/pubmed/15053126.

7. Pylatiuk C, Schulz S, Döderlein L. Results of an Internet survey of myoelectric prosthetic hand users. Prosthet Orthot Int. 2007;31(4):362-370. doi:10.1080/03093640601061265.

8. Light CM, Chappell PH, Kyberd PJ. Establishing a standardized clinical assessment tool of pathologic and prosthetic hand function: normative data, reliability, and 
validity. Arch Phys Med Rehabil. 2002;83(6):776-783.

http://www.ncbi.nlm.nih.gov/pubmed/12048655. Accessed April 3, 2014.

9. Hermansson LM, Fisher AG, Bernspång B, Eliasson A-C. Assessment of capacity for myoelectric control: a new Rasch-built measure of prosthetic hand control. J Rehabil Med. 2005;37(3):166-171. doi:10.1080/16501970410024280.

10. Jones LA. The assessment of hand function: a critical review of techniques. $J$ Hand Surg Am. 1989;14(2 Pt 1):221-228. http://www.ncbi.nlm.nih.gov/pubmed/2649546.

11. Hussaini A, Kyberd P. Refined clothespin relocation test and assessment of motion. Prosthet Orthot Int. 2017;41(3):294-302. doi:10.1177/0309364616660250.

12. Bouwsema H, Kyberd PJ, Hill W, van der Sluis CK, Bongers RM. Determining skill level in myoelectric prosthesis use with multiple outcome measures. J Rehabil Res Dev. 2012;49(9):1331-1348. http://www.ncbi.nlm.nih.gov/pubmed/23408215. Accessed March 11, 2014.

13. Gaine WJ, Smart C, Bransby-Zachary M. Upper limb traumatic amputees. Review of prosthetic use. J Hand Surg Br. 1997;22(1):73-76. http://www.ncbi.nlm.nih.gov/pubmed/9061532.

14. Hill W, Stavdahl Ø, Hermansson LN, Kyberd P, Swanson S, Hubbard S. Functional Outcomes in the WHO-ICF Model: Establishment of the Upper Limb Prosthetic Outcome Measures Group. JPO J Prosthetics Orthot. 2009;21(2):115-119. doi:10.1097/JPO.0b013e3181a1d2dc.

15. Wright F. Measurement of functional outcome with individuals who use upper extremity prosthetic devices: current and future directions. J Prosthetics Orthot. 2006;18(2):46-56.

16. Vujaklija I, Roche AD, Hasenoehrl T, et al. Translating Research on Myoelectric Control into Clinics-Are the Performance Assessment Methods Adequate? Front Neurorobot. 2017;11. doi:10.3389/fnbot.2017.00007. 
17. Burger H, Brezovar D, Vidmar G. A comparison of the University of New Brunswick Test of Prosthetic Function and the Assessment of Capacity for Myoelectric Control. Eur J Phys Rehabil Med. 2014;50(4):433-438.

http://www.ncbi.nlm.nih.gov/pubmed/24476807.

18. Resnik L, Adams L, Borgia M, et al. Development and evaluation of the activities measure for upper limb amputees. Arch Phys Med Rehabil. 2013;94(3):488-494.e4. doi:10.1016/j.apmr.2012.10.004.

19. Biddiss EA, Chau TT. Upper limb prosthesis use and abandonment: a survey of the last 25 years. Prosthet Orthot Int. 2007;31(3):236-257. doi:10.1080/03093640600994581.

20. Lyle RC. A performance test for assessment of upper limb function in physical rehabilitation treatment and research. Int J Rehabil Res. 1981;4(4):483-492. http://www.ncbi.nlm.nih.gov/pubmed/7333761. Accessed March 11, 2014.

21. Kyberd PJ, Murgia A, Gasson M, et al. Case studies to demonstrate the range of applications of the Southampton Hand Assessment Procedure Practice evaluation. 2009;72(September 2007):212-218.

22. Mathiowetz V, Volland G, Kashman N, Weber K. Adult norms for the Box and Block Test of manual dexterity. Am J Occup Ther Off Publ Am Occup Ther Assoc. 1985;39(6):386-391. doi:10.5014/ajot.39.6.386.

23. Yozbatiran N, Der-Yeghiaian L, Cramer SC. A standardized approach to performing the action research arm test. Neurorehabil Neural Repair. 22(1):78-90. doi:10.1177/1545968307305353.

24. Miller LA, Swanson S. Summary and Recommendations of the Academy's State of the Science Conference on Upper Limb Prosthetic Outcome Measures. In: American Academy of Orthotists and Prosthetists.; 2009:83-89.

25. Salminger S, Sturma A, Roche AD, et al. Functional and Psychosocial Outcomes of Hand Transplantation Compared with Prosthetic Fitting in Below-Elbow Amputees: A 
Multicenter Cohort Study. PLoS One. 2016;11(9):e0162507. doi:10.1371/journal.pone.0162507.

26. Ninkovic M, Weissenbacher A, Gabl M, et al. Functional outcome after hand and forearm transplantation: what can be achieved? Hand Clin. 2011;27(4):455-465, viii ix. doi:10.1016/j.hcl.2011.08.005.

27. Bernardon L, Gazarian A, Petruzzo P, et al. Bilateral hand transplantation: Functional benefits assessment in five patients with a mean follow-up of 7.6 years (range 4-13 years). J Plast Reconstr Aesthet Surg. 2015;68(9):1171-1183. doi:10.1016/j.bjps.2015.07.007.

28. Kejlaa GH. Consumer concerns and the functional value of prostheses to upper limb amputees. Prosthet Orthot Int. 1993;17(3):157-163.

http://www.ncbi.nlm.nih.gov/pubmed/8134275. 


\section{Tables}

Table I: Patients demographics

Table II: Function outcomes (Action Research Arm Test (ARAT); Southampton Hand Assessment Procedure (SHAP); Clothespin Relocation Test (CPRT); Box and Blocks Test $(\mathrm{BBT})$ 


\begin{tabular}{|c|c|c|c|c|c|c|c|}
\hline Patients & Sex & Age & $\begin{array}{c}\text { Age at } \\
\text { amputation }\end{array}$ & $\begin{array}{c}\text { Side of } \\
\text { amputation }\end{array}$ & $\begin{array}{l}\text { Dominant } \\
\text { side prior to } \\
\text { amputation }\end{array}$ & $\begin{array}{l}\text { Prosthetic } \\
\text { experience } \\
\text { in years }\end{array}$ & History \\
\hline 1 & $\mathrm{~m}$ & 39 & 28 & right & right & 10 & Traumatic \\
\hline 2 & $\mathrm{~m}$ & 24 & 21 & right & right & 2.5 & Traumatic \\
\hline 3 & $\mathrm{~m}$ & 50 & 45 & right & right & 2.5 & Traumatic \\
\hline 4 & $\mathrm{~m}$ & 33 & 24 & left & left & 8 & Traumatic \\
\hline 5 & $\mathrm{~m}$ & 32 & 30 & left & right & 2.5 & Traumatic \\
\hline 6 & $\mathrm{~m}$ & 21 & 0 & right & left & 10 & Congenital \\
\hline 7 & $\mathrm{~m}$ & 28 & 25 & right & right & 3 & Traumatic \\
\hline 8 & $\mathrm{~m}$ & 26 & 25 & right & right & 1 & Traumatic \\
\hline 9 & $\mathrm{f}$ & 37 & 20 & right & right & 16 & Traumatic \\
\hline 10 & $\mathrm{~m}$ & 47 & 45 & left & right & 1.5 & Traumatic \\
\hline 11 & $\mathrm{~m}$ & 28 & 22 & left & right & 6 & Traumatic \\
\hline 12 & $\mathrm{~m}$ & 28 & 12 & right & right & 15 & Tumor \\
\hline 13 & $\mathrm{~m}$ & 44 & 27 & right & right & 1 & Traumatic \\
\hline 14 & $\mathrm{~m}$ & 56 & 20 & left & right & 25 & Traumatic \\
\hline 15 & $\mathrm{~m}$ & 37 & 33 & left & left & 3.5 & Traumatic \\
\hline 16 & $\mathrm{~m}$ & 31 & 26 & right & right & 4.5 & Traumatic \\
\hline 17 & $\mathrm{~m}$ & 44 & 41 & right & right & 3 & Traumatic \\
\hline Mean & & 35.59 & 26.12 & & & 6.76 & \\
\hline SD & & 9.89 & 11.20 & & & 6.62 & \\
\hline
\end{tabular}




\begin{tabular}{|c|c|c|c|c|c|}
\hline Patients & ARAT & BBT & CPRT & SHAP & $\begin{array}{c}\text { Wearing } \\
\text { time in } \\
\text { hours }\end{array}$ \\
\hline 1 & 44 & 26.33 & 15.10 & 85 & 12 \\
\hline 2 & 36 & 27.00 & 17.47 & 83 & 11 \\
\hline 3 & 35 & 21.00 & 20.61 & 69 & 7 \\
\hline 4 & 36 & 24.00 & 14.54 & 72 & 13,5 \\
\hline 5 & 36 & 28.00 & 19.55 & 70 & 13 \\
\hline 6 & 35 & 17.00 & 21.74 & 72 & 12 \\
\hline 7 & 38 & 13.33 & 35.87 & 60 & 12 \\
\hline 8 & 32 & 15.00 & 30.94 & 59 & 18 \\
\hline 9 & 30 & 21.00 & 26.75 & 46 & 13,5 \\
\hline 10 & 32 & 15.00 & 19.28 & 53 & 9 \\
\hline 11 & 42 & 31.00 & 17.58 & 83 & 14 \\
\hline 12 & 30 & 14.67 & 22.32 & 53 & 9 \\
\hline 13 & 29 & 13.67 & 42.76 & 37 & 10 \\
\hline 14 & 32 & 26.33 & 20.90 & 55 & 11 \\
\hline 15 & 35 & 22.00 & 19.32 & 75 & 13 \\
\hline 16 & 42 & 24.00 & 18.96 & 78 & 13 \\
\hline 17 & 32 & 16.00 & 20.01 & 57 & 11 \\
\hline Mean & 35.06 & 20.90 & 22.57 & 65.12 & 11.88 \\
\hline $\mathrm{SD}$ & 4.42 & 5.74 & 7.50 & 13.95 & 2.47 \\
\hline
\end{tabular}

